\title{
Total solar irradiance as measured by the SOVAP radiometer onboard PICARD
}

\author{
Mustapha Meftah ${ }^{1, *}$, André Chevalier ${ }^{2}$, Christian Conscience ${ }^{2}$, and Stijn Nevens ${ }^{2}$ \\ 1 University of Paris-Saclay, University of Paris VI, CNRS, LATMOS, 11 Boulevard d'Alembert, 78280 Guyancourt, France \\ ${ }^{*}$ Corresponding author: Mustapha.Meftah@latmos.ipsl.fr \\ 2 Royal Meteorological Institute of Belgium, 3 Avenue Circulaire, 1180 Uccle, Belgique
}

Received 27 January 2016 / Accepted 15 August 2016

\begin{abstract}
From the SOlar VAriability PICARD (SOVAP) space-based radiometer, we obtained a new time series of the total solar irradiance (TSI) during Solar Cycle 24. Based on SOVAP data, we obtained that the TSI input at the top of the Earth's atmosphere at a distance of one astronomical unit from the Sun is $1361.8 \pm 2.4 \mathrm{~W} \mathrm{~m}^{-2}(1 \sigma)$ representative of the 2008 solar minimum period. From 2010 to 2014, the amplitude of the changes has been of the order of $\pm 0.1 \%$, corresponding to a range of about $2.7 \mathrm{~W} \mathrm{~m} \mathrm{~m}^{-2}$. To determine the TSI from SOVAP, we present here an improved instrument equation. A parameter was integrated from a theoretical analysis that highlighted the thermo-electrical non-equivalence of the radiometric cavity. From this approach, we obtained values that are lower than those previously provided with the same type of instrument. The results in this paper supersede the previous SOVAP analysis and provide the best SOVAP-based TSI-value estimate and its temporal variation.
\end{abstract}

Key words. Sun - Total Irradiance - Solar activity - Solar Cycle - Spacecraft

\section{Introduction}

Solar activity (i.e. total solar irradiance (TSI), solar magnetic field, ultraviolet radiation, etc.) is an important contributor to natural climate variability. Thus, the long-term monitoring of the Essential Climate Variable (ECV) TSI is important in order to understand the change of climate with time. Since 1978 , the absolute value of the TSI and its temporal variation have been measured from space. Prior to the launch of the SOlar Radiation and Climate Experiment (SORCE) spacecraft in January 2003, the consensus absolute value of the TSI was close to $1365.4 \mathrm{~W} \mathrm{~m}^{-2}$. The Total Irradiance Monitor (TIM) instrument onboard SORCE measured deviating values around $1361 \mathrm{~W} \mathrm{~m}^{-2}$ (Kopp \& Lean 2011). This result was independently obtained by the PREcision MOnitor Sensor (PREMOS) instrument onboard PICARD (Schmutz et al. 2013). Before its launch, PREMOS had been calibrated relative to the cryogenic Total solar irradiance Radiometer Facility (TRF) of the Laboratory for Atmospheric and Space Physics (LASP). PREMOS was also calibrated against the World Radiometric Reference (WRR) to get a consistent picture. The measurements of the SOlar VAriability PICARD (SOVAP) space-based radiometer yielded a TSI value quite close to TIM and PREMOS results (Meftah et al. 2014). To obtain this value, the instrument equation was updated accounting for the thermo-electrical non-equivalence of the SOVAP radiometric cavity. Moreover, the 35-year Active Cavity Radiometer Irradiance Monitor (ACRIM) TSI satellite composite time series has been revised (Willson 2014) using algorithm updates and corrections to ACRIMSAT/ACRIM3 results for scattering and diffraction derived from testing with the TRF. Thus, ACRIM3 has lowered its space TSI value after the comparison of an ACRIM engineering model with the cryogenic TRF. Finally, the Variability of Irradiance and
Gravity Oscillations (VIRGO) experiment (Fröhlich et al. 1995) onboard the Solar and Heliospheric Observatory (SoHO) provided a new absolute value of the TSI (new scale) that is $0.42 \%$ lower than the original scale $\left(\sim 1365.4 \mathrm{~W} \mathrm{~m}^{-2}\right)$. The VIRGO results (VIRGO new scale, VIRGO-PMO6 V and VIRGO-DIARAD) are available at $\mathrm{ftp}$ //ftp.pmodwrc.ch/pub/data/irradiance/virgo/TSI/.

Space observations with these experiments are consistent with a mean TSI value of $1361 \pm 1 \mathrm{~W} \mathrm{~m}^{-2}(2 \sigma)$, which is a representative absolute value of the 2008 solar minimum period. Despite the advent of the space era, the question addressing the absolute TSI determination remains open. Indeed, the debate about absolute TSI determination remains in a large way limited to the determination of realistic uncertainties. In 2005, it was suggested (Butler et al. 2008) that every TSI team should critically investigate its own instrument, and that on-ground comparisons between radiometers in power and in irradiance modes should be made. The latter recommendation has led different teams to take their radiometers to the LASP/TRF facility, in which a radiometer under test can be compared in vacuum for power and irradiance measurement with a reference cryogenic radiometer. We point out that the SOVAP instrument (Conscience et al. 2011) was not calibrated at the TRF facility.

In this paper, we report on the TSI measurements of SOVAP, which were carried out during the PICARD mission from July 2010 to March 2014. We provide our best estimate of the absolute value of the TSI as seen by SOVAP. We correct the determination of the SOVAP thermo-electrical nonequivalence between the right and the left cavity during deep space measurements. Finally, we provide a SOVAP TSI composite that includes all measurement modes normalised to the mean TSI of the nominal modes. 


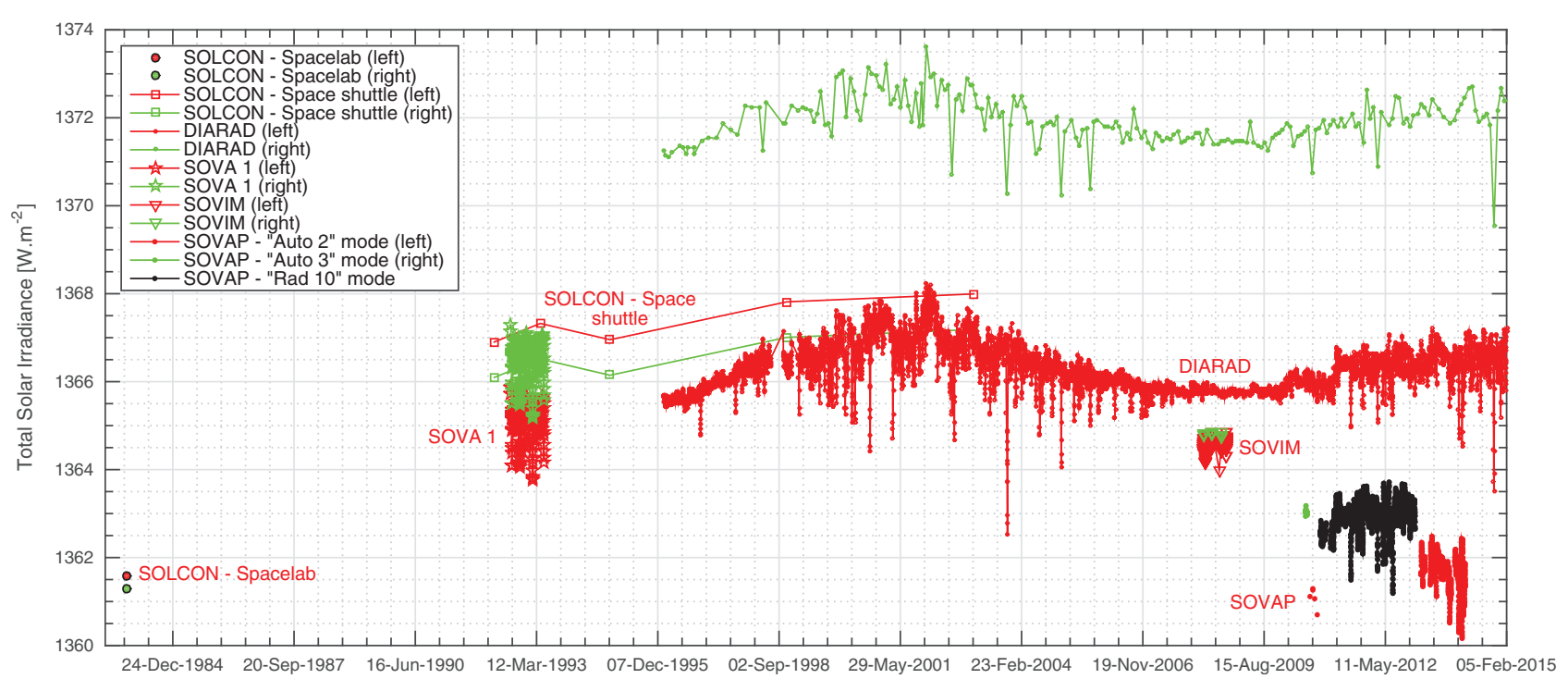

Fig. 1. TSI published time series for the left and right channels of the main space-based TSI radiometers developed by the RMIB: the SOLar CONstant instrument (SOLCON) on Spacelab and onboard the space shuttle, the Solar Variations (SOVA 1) experiment onboard European Retrievable Carrier (EURECA), VIRGO-DIARAD onboard SoHO, the Sun Monitoring on the External Payload Facility of Columbus-SOlar Variable and Irradiance Monitor (Solar-SOVIM). New TSI time series of SOVAP onboard PICARD are displayed for the different modes with the correction for non-equivalence included.

\section{TSI observations by RMIB since 1983}

The Royal Meteorological Institute of Belgium (RMIB) has in its 33-year space history, since Spacelab in November 1983, launched six different TSI instruments of the DIfferential Absolute RADiometer (DIARAD) type on 11 space flights. Figure 1 displays the long-term TSI time series of the main space-based TSI radiometers developed by RMIB. Only the SOVAP TSI data were corrected for the thermo-electrical non-equivalence of the cavity (Meftah et al. 2014).

The DIARAD instrument consists of two electrical substitution radiometers driven by a servo-system. The radiometers are symmetrical cylindrical cavities, mounted side by side on a common heat sink. Both cavities see the same thermal environment through accurately known circular precision apertures (Conscience et al. 2011). Part of the light reflected from the precision aperture illuminates the baffle and, especially in vacuum, heats it. Both effects increase the measured signal as scattered light and infrared radiation from the baffle enter the cavities. For all of the classical radiometers, this effect has been extensively studied at the TRF facility, where corrections for PREMOS and ACRIM3 were determined. This effect is negligible in the DIARAD type radiometers due to the ingenious construction of the primary aperture as a spherical mirror which focuses this undesired incoming light into the centre of the front-most view-limiting aperture and out of the instrument. On the other hand, the DIARAD design has a fundamental limitation due to a distributed thermal impedance on the surface of the sensor and an electrical heater which covers a larger area than the solar beam. This effect is present in all RMIB radiometers, and a specific correction for each radiometer is needed to correct formerly obtained results.

In 1983, a cavity-type absolute radiometer was flown on Spacelab where a TSI value of $1361.5 \pm 2.3 \mathrm{~W} \mathrm{~m}^{-2}$ (at one standard deviation $(1 \sigma)$ ) was obtained (Crommelynck et al. 1987), which is atypical compared to other RMIB results.
The Spacelab radiometer has a different thermo-electric sensor, which in hindsight may explain why it measured a much more accurate value of the TSI. The thermal conductivity of the faces of this instrument's sensors is different from most RMIB radiometers, while the size of the heater elements is the same. The thermo-mechanical properties of the Spacelab cavity cylinders and their interfaces to the sensor are also different. These results may explain the difference of this radiometer relative to the others mentioned in Crommelynck et al. (1987). This needs further investigation.

One of the RMIB radiometers, VIRGO-DIARAD (Dewitte et al. 2004), acquired TSI measurements as part of the VIRGO experiment (Fröhlich et al. 1995, 1997) onboard SoHO from 1996 until the present, which represents one of the longest TSI-measurement time series. As a reminder, the DIARAD radiometer is part of VIRGO together with two PMO6V-type radiometers as discussed in Section 1. Having two radiometers of different designs may help to understand the behaviour of the radiometers in space and hence provide a more reliable estimate of TSI from a combination of the results from both. This combination is called VIRGO TSI (new scale, version 6).

Our understanding of the long-term behaviour of spacebased TSI radiometers has increased substantially. The RMIB uses the measurements of DIARAD as a stand-alone instrument, which could be problematic because this type of radiometer could show a non-exposure-dependent sensitivity change. This effect cannot be corrected internally by comparing with a less exposed radiometer. Indeed, it is important to characterise the long-term behaviour especially for the 20 -year record of VIRGO. For long-term characterisations of SOVAP, in this paper we use the RMIB VIRGO-DIARAD TSI values to compare these two radiometers based on the same technology and methods. The new scale of VIRGO values, which are based on both instruments (VIRGO-PMO6V and VIRGO-DIARAD), are consistent within uncertainties with the SOVAP absolute value of the TSI. 
M. Meftah et al.: TSI time series during Solar Cycle 24

Table 1. SOVAP radiometer parameters' definition and typical values for the "Rad 10" mode.

\begin{tabular}{llc}
\hline \hline Parameters & \multicolumn{1}{c}{ Definition } & Typical values \\
\hline$P_{c l, l}-P_{o, r}$ & Difference in electrical power applied to the cavities & $\sim 0.1056 \mathrm{~W}$ \\
$C_{1}$ & Correction to take into account the heating effects by the wires & $5.9 \times 10^{-6} \mathrm{~W}$ \\
$C_{2}$ & Correction for the accuracy of the servo-system (almost negligible) & $1.2 \times 10^{-6}$ \\
$C_{3}^{\prime}$ & Correction of the thermal radiative effects of the left shutter & $\sim 0.405 \mathrm{~W} \mathrm{~m}{ }^{-2}$ \\
$C_{4}$ & Correction of the thermal radiative effects of the mirror above the cavity & $\sim-0.175 \mathrm{~W} \mathrm{~m}^{-2}$ \\
$C_{5}$ & Total correction factor for the optical effects (diffraction and scattering) & 0.999447805 \\
$\alpha_{\text {eff, } r}$ & Efficiency of the right cavity & 0.9987 \\
$A_{r}$ at $20{ }^{\circ} \mathrm{C}$ & Measured accurate aperture area at $20{ }^{\circ} \mathrm{C}$ & $7.876 \times 10^{-5} \mathrm{~m}^{2}$ \\
$\Delta_{r}$ & Aperture area (AA) correction factor - expansion of the mirror & $\sim 1.9 \times 10^{-8}$ \\
$\Delta_{r}^{\prime}$ & AA correction factor - expansion of the radiometer & $\sim 5.4 \times 10^{-8}$ \\
$\Delta_{r}^{\prime \prime}$ & AA correction factor - deformation during mechanical clamping & $\sim 0$ \\
$\cos \theta$ & Solar pointing angle of the radiometer & $\sim 1$ \\
$\Delta P_{o, r}$ & Thermo-electrical non-equivalence of the active cavity while open & $\sim 0.3 \times 10^{-3} \mathrm{~W}$ \\
$K_{\mathrm{Rad} 10}$ & Thermo-electrical non-equivalence between the right and the left cavity & $\sim 2.83 \times 10^{-3} \mathrm{~W}$ \\
\hline
\end{tabular}

\section{SOVAP operations summary}

DIARAD is an electrically calibrated cavity radiometer, based on a differential heat-flux meter. The main advantage is that both cavities can be used for solar measurements and their arrangement views the same thermal environment. In the nominal operation modes, the radiometric equation for each cavity (left or right) is given in Eq. (2) with all the details described by Meftah et al. (2014). The modes are called "Auto 2" and "Auto 3" for left and right operations, respectively. For each mode, the sampling period is three minutes (a sequence of $90 \mathrm{~s}$ open and $90 \mathrm{~s}$ closed) and provides an instrument demonstrated repeatability around $0.1 \mathrm{~W} \mathrm{~m}^{-2}$. The "Auto 2" mode was intended to track on-orbit degradation of the primary right cavity ("Auto 3" mode) via infrequent exposure of the left cavity (roughly every month). The left channel provided a TSI measurement with the lowest absolute uncertainty $\left( \pm 1.39 \mathrm{~W} \mathrm{~m}^{-2}\right)$ as shown by Meftah et al. (2014).

The "Auto 3" mode was used intermittently from July 22, 2010 until August 28, 2010. Few measurements were achieved with the SOVAP-DIARAD right channel ("Auto 3" mode). The "Auto 2" mode was used to measure the TSI absolute level as well as the TSI variability for half an hour every month from August 28, 2010 to October 27, 2010.

Early on in the mission, the "Auto 3" mode was abandoned due to a failure of the right shutter mechanism that left the cavity permanently open. A change in the operation was needed where an alternative mode was used. During this new "Rad 10" mode, the right shutter is permanently open and the left shutter is kept closed. This mode was used starting with the first occultation period on the 18th of November 2010 (after an update of the flight software). Thus, the SOVAP right shutter was permanently open and the left shutter was kept closed from November 2010 until January 2013. The main difference in the radiometric Eq. (3) of the "Rad 10" mode is that the power fed to the left cavity in closed state $\left(P_{c l, l}\right)$ is not representative for the right channel and a correction called $K_{\mathrm{Rad} 10}$ has to be introduced. Moreover, the shutter correction has to be changed to only take into account the emission of the cavity to deep space instead of the difference of this to the shutter emission in the closed position. This shutter correction now is only dependent on the temperature of the cavity which is known. Furthermore, we need to calibrate $K_{\mathrm{Rad} 10}$ and this is done by measuring deep space in "Rad 10 " mode which is then the only unknown in the equation. The "Rad 10" mode had the advantage of increasing the measurement time resolution. In fact, SOVAP measurements were organised in packets of $90 \mathrm{~s}$, containing several frames of $10 \mathrm{~s}$ measurements each. TSI acquisition was made at seconds 20, 40, 70, 80 and 90. In the "Rad 10" mode measurements, the active cavity is always in thermo-electrical equilibrium. Therefore, all TSI measurements at seconds 20, 40, 70, 80 and 90 can be used and no $C_{6}$ correction (dynamic nonequivalence between electrical and radiative response) is needed as shown in Eq. (3). In DIARAD design, the sensors are in the feed-back loop, but the cylindrical parts of the cavities aren't. We determine the $C_{6}$ parameter in flight by comparison of the response of the normal sampling time of $3 \mathrm{~min}(90 \mathrm{~s}$ open and $90 \mathrm{~s}$ closed) with a doubled sampling time of $6 \min (180 \mathrm{~s}$ open and $180 \mathrm{~s}$ closed) to ensure the system reaches thermal equilibrium.

In early 2013, it was decided that the PICARD space mission would end at the beginning of March 2014. Thus, it was no longer necessary to protect the integrity of the left cavity for ageing determination. Normally, the degradation is tracked with the cavity that is less exposed to solar radiation in order to minimise the degradation due to solar UV radiation. We tried and succeeded to close the right shutter and then turn on the "Auto 2" mode and it was then used from February 2013 until March 2014. This operating mode was intensively used after several months in space.

\section{SOVAP TSI equation and measurement uncertainty}

TSI is defined as the power of the incoming solar electromagnetic radiation per unit area at one astronomical unit (1 au). The SOVAP TSI Eq. (1) follows this convention

$$
\operatorname{TSI}_{(i=r, l)}=\operatorname{SI}_{(i)} \times \frac{z^{2}}{(1 \mathrm{au})^{2} \times\left(1-\frac{\partial z}{\partial t} \times \frac{1}{c}\right)^{2}},
$$

where $\operatorname{TSI}_{(i=r, l)}$ is the total solar irradiance $\left(\mathrm{W} \mathrm{m}^{-2}\right)$ of the right cavity $(r)$ or the left cavity $(l)$ at 1 au. $\mathrm{SI}_{(i)}$ is the solar irradiance $\left(\mathrm{W} \mathrm{m}^{-2}\right)$ measured at the spacecraft's distance from the Sun, $z$ is the distance between the PICARD spacecraft and the Sun $(\mathrm{km})$, the astronomical unit is equal to $149,597,870.700 \mathrm{~km}, c$ is the speed of light $(299,792.458 \mathrm{~km}$ per second) and $t$ is the time (second).

\subsection{SOVAP TSI equation - "Auto 2" and "Auto 3" modes}

$\mathrm{SI}_{(i)}$ is derived from the measurement of the electrical power supplied to the active cavity of the radiometer to maintain this 


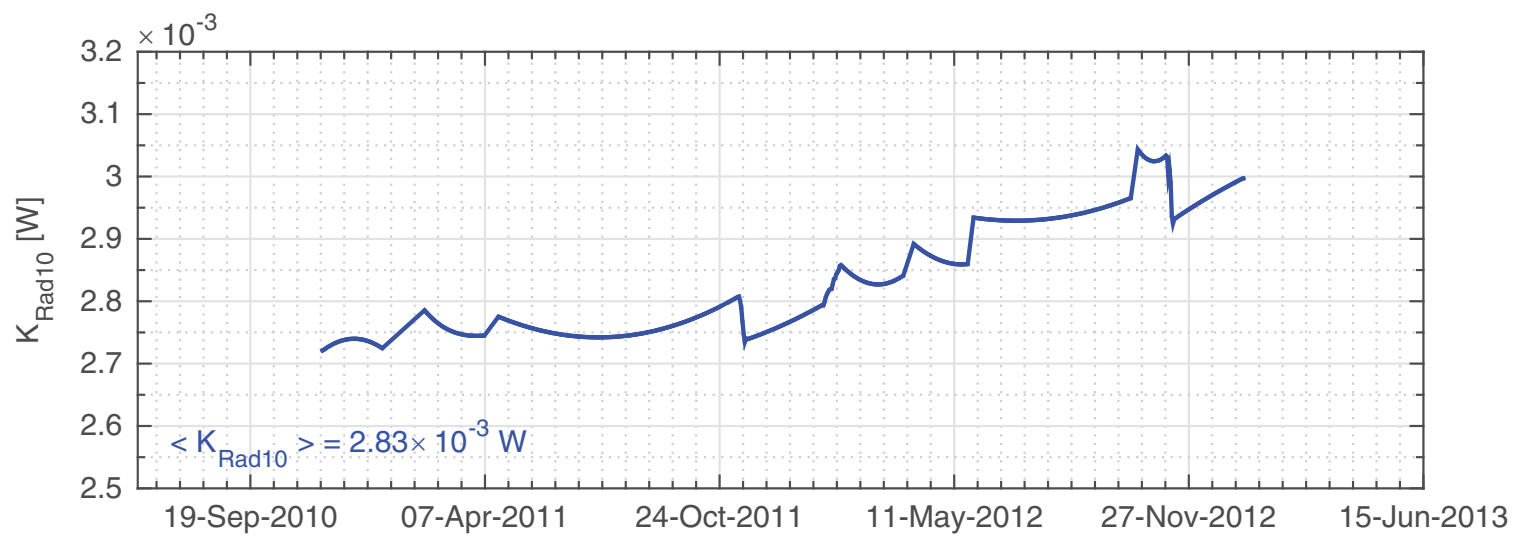

Fig. 2. The blue curve corresponds to the "zero solar flux" on the open cavity $\left(K_{\text {Rad10 }}\right)$. The PICARD spacecraft is in a sun-synchronous orbit and goes into Earth eclipse seasons from November to February. During these eclipses, the spacecraft passes into the penumbra (solar flux through the Earth atmosphere) and the umbra of the Earth that implies high variability of $P_{c l, l}-P_{o, r}$.

cavity with the same heat sink flux in the open and closed states as described in more detail by Meftah et al. (2014). The measured solar irradiance for the nominal modes is given by Eq. (2), where the constants are defined in Table 1 except for $C_{6}$ that is not needed in the "Rad 10 " mode and was explained earlier (end of Sect. 3).

$$
\begin{aligned}
& \mathrm{SI}_{(i=r, l)}= \\
& C_{5} \times \frac{\left(\left(\overline{P_{c l, i}}-P_{o, i}\right)-\Delta P_{o, i}+C_{1}+C_{6}\right) \times\left(1-C_{2}\right)}{\alpha_{\mathrm{eff}, i} \times A_{i} \times\left(1+\Delta_{i}+\Delta_{i}^{\prime}+\Delta_{i}^{\prime \prime}\right) \times \cos \theta} \\
& \quad+C_{3}+C_{4} .
\end{aligned}
$$

The estimated uncertainty on the TSI absolute determination for the "Auto 2 " mode is $\pm 1.4 \mathrm{Wm}^{-2}$ at $1 \sigma$ and for the "Auto 3" mode the estimated uncertainty is $\pm 1.5 \mathrm{Wm}^{-2}$ at $1 \sigma$ (Meftah et al. 2014).

\subsection{SOVAP TSI equation - "Rad 10" mode}

To calculate the TSI in this case, a slightly changed instrument equation is required where both channels are used to determine the SOVAP TSI. Solar Irradiance $\left(\mathrm{SI}_{(R 10)}\right)$ for the SOVAP right cavity during the "Rad 10 " mode is derived from the measurement of the difference between the electrical power supplied to the closed cavity (left) and the electrical power supplied to the open cavity (right).

$$
\begin{aligned}
& \mathrm{SI}_{(R 10)}= \\
& C_{5} \times \frac{\left(\left(P_{c l, l}-P_{o, r}\right)-\Delta P_{o, r}-K_{\mathrm{Rad} 10}+C_{1}\right) \times\left(1-C_{2}\right)}{\alpha_{\mathrm{eff}, r} \times A_{r} \times\left(1+\Delta_{r}+\Delta_{r}^{\prime}+\Delta_{r}^{\prime \prime}\right) \times \cos \theta} \\
& \quad+\mathrm{C}_{3}^{\prime}+C_{4} .
\end{aligned}
$$

where $P_{c l, l}$ is the electrical power (W) fed to the left cavity in closed state of the shutter, $P_{o, r}$ is the electrical power $(\mathrm{W})$ fed to the right cavity in open state of the shutter and $K_{\mathrm{Rad} 10}$ is the thermo-electrical non-equivalence between the right and the left cavity of the SOVAP radiometer (obtained during space calibration). The definition of the parameters of Eq. (3) with typical values is provided in Table 1. Each parameter is described in more detail in the SOVAP instrumental paper (Conscience et al. 2011), except $C_{3}^{\prime}$ that is temperature dependent and is given in Table 1 for the mean temperature of the closed shutter.

The $K_{\mathrm{Rad} 10}$ parameter is obtained during space calibration (stellar calibration and Earth eclipse seasons) where the right cavity was not exposed to sunlight. The evolution of the $K_{\text {Rad10 }}$ parameter is displayed in Figure 2 . The $K_{\mathrm{Rad} 10}$ parameter changes over time and stems from a changing difference between the two channels. The physical explanations for the change in $K_{\mathrm{Rad} 10}$ over time could be an overall warming up of the instrument/heat sink, a modification of the main characteristics of the thermo-electric sensor (heat-flux transducer), a creep of the thermo-electric sensor that is mounted between the cavity and the heat sink (indium thermal interface material), and probably an evolution of the specific torque of the attachment of the thermo-electric sensor. $K_{\mathrm{Rad} 10}$ evolves between $2.7 \times 10^{-3}$ and $3.1 \times 10^{-3} \mathrm{~W}$. Thus, the SOVAP TSI variability time series ("Rad 10" mode) is shown in Figure 3 using Eq. (3) with the different parameters of the Table 1 such as $K_{\text {Rad10. }}$. Uncertainties of the absolute determination of TSI for the "Rad 10" mode are given in Table 2 in parts per million (ppm) and in $\mathrm{W} \mathrm{m}^{-2}$. Electrical chain (general effects, transfer of calibration and ground parasitic resistance) uncertainties take into account the two cavities (left and right). Thus, the "Rad 10" mode uncertainty is of the order of $\pm 1.7 \mathrm{~W} \mathrm{~m}^{-2}(1 \sigma)$, which is higher than nominal modes due to the lack of shuttering.

\section{SOVAP TSI time series, results and discussion}

The SOVAP radiometer made TSI measurements from July 2010 to March 2014. These measurements are compared to TSI time series of existing data and may help to resolve some of the differences in existing long-term time series of TSI. Thus, comparisons were carried out between the Level 2 SOVAP data, VIRGO-DIARAD measurements, TIM measurements and PREMOS measurements. Moreover, a comparison between SOVAP measurements and Spectral And Total Irradiance REconstruction for the Satellite era (SATIRE-S) semi-empirical model (Yeo et al. 2014) was performed. The TSI levels for main operation modes of 


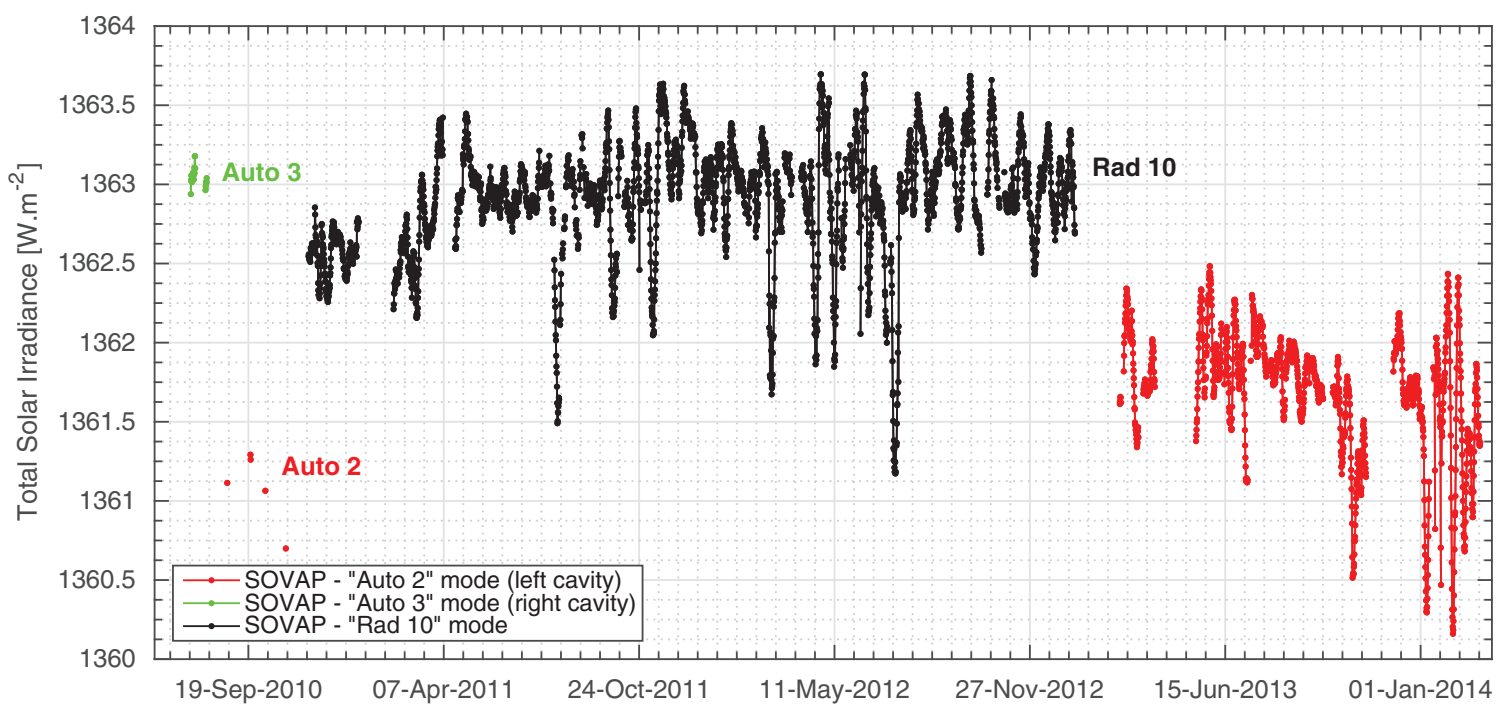

Fig. 3. The red curve corresponds to the "Auto 2" sequence of measurements (left channel). The green curve corresponds to the "Auto 3 " sequence of measurements (right channel). The black curve corresponds to the "Rad 10" sequence of measurements (right channel with the left channel as reference).

Table 2. Uncertainties of the TSI measurements for the "Rad 10" mode.

\begin{tabular}{lrc}
\hline \hline Parameters of the right channel & $(\mathrm{ppm})$ & $\left.(\mathrm{Wm})^{-2}\right)$ \\
\hline Wires heating effect $C_{1}$ & 1.5 & 0.002 \\
Accuracy of the servo-system $C_{2}$ & 1.2 & 0.002 \\
Infrared emission of the shutter $C_{3}^{\prime}$ & 39.0 & 0.053 \\
Infrared emission of the mirror $C_{4}$ & 19.0 & 0.026 \\
Optical effects $C_{5}$ & 110.0 & 0.150 \\
Absorption factor $\alpha_{\text {eff, } r}$ & 190.0 & 0.259 \\
Precision aperture $A_{r}$ at $2{ }^{\circ} \mathrm{C}$ & 117.0 & 0.159 \\
Thermal effects $\Delta_{r}$ & 30.0 & 0.041 \\
Thermo-mechanical effect $\Delta_{r}^{\prime}$ & 250.0 & 0.341 \\
Fixation of the aperture $\Delta_{r}^{\prime \prime}$ & 250.0 & 0.341 \\
Thermo-mechanical sliding cos $\theta$ & 6.0 & 0.008 \\
Thermo-electrical non-equivalence $\Delta P_{o}$ & 837.0 & 1.140 \\
Thermo-electrical non-equivalence $K_{\mathrm{Rad} 10}$ & 280.0 & 0.381 \\
Thermal radiative effects of the baffle & 13.0 & 0.018 \\
Electrical chains (general effects) & 732.6 & 0.998 \\
Electrical chains (transfer of calibration) & 175.4 & 0.239 \\
Electrical chains (ground parasitic resistance) & 284.2 & 0.387 \\
Doppler effect & 50.0 & 0.068 \\
Mean quadratic uncertainty $(\sigma=1)$ & 1272.6 & 1.733 \\
\hline
\end{tabular}

SOVAP-DIARAD are presented in Figure 3, which are the same data as in Figure 1. SOVAP instrument equations (see Sect. 4) were used to obtain these levels. From Eq. (3), we get the evolution over time of the SOVAP "Rad 10" TSI.

\subsection{TSI absolute value from SOVAP nominal modes - "Auto 2" and "Auto 3"}

In 2010, the first measurements made by the SOVAP radiometer correspond to a state where the instrument was as characterised on ground. The radiometer had not yet suffered degradation due to the effects of the space environment and radiation. A combination of solar irradiation and instrumental contamination can cause polymerisation of organic material and, subsequently, irreversible deposition of this material on the radiometer. Moreover, the black paint used in the cavities degrades due to radiation. These first measurements are fundamental with respect to the determination of the TSI absolute value. In these conditions, the "Auto 2" SOVAP measurements give a TSI value of $1361.1 \mathrm{~W} \mathrm{~m}^{-2}$ with an uncertainty of $\pm 1.4 \mathrm{~W} \mathrm{~m}^{-2}(1 \sigma)$. Similarly, the "Auto 3 " SOVAP measurements provide a TSI value of $1363.0 \pm 1.5 \mathrm{~W} \mathrm{~m}^{-2}(1 \sigma)$. By normalising these measurements using VIRGO-DIARAD TSI (data extrapolation) with respect to the 2008 solar minimum period, the "Auto 2" SOVAP TSI is close to 1360.9 $\mathrm{W} \mathrm{m}^{-2}$ and the "Auto 3" SOVAP TSI is close to $1362.8 \mathrm{~W} \mathrm{~m}^{-2}$. The two completely characterised measurements differ by $\sim 2 \mathrm{~W} \mathrm{~m}^{-2}$, where the reproducibility of the thermo-electric sensor characteristics and its integration has a fundamental impact on the differences. Thus, the most conservative approach to determine the absolute value of the SOVAP TSI is to take into account both channels of the radiometer. 


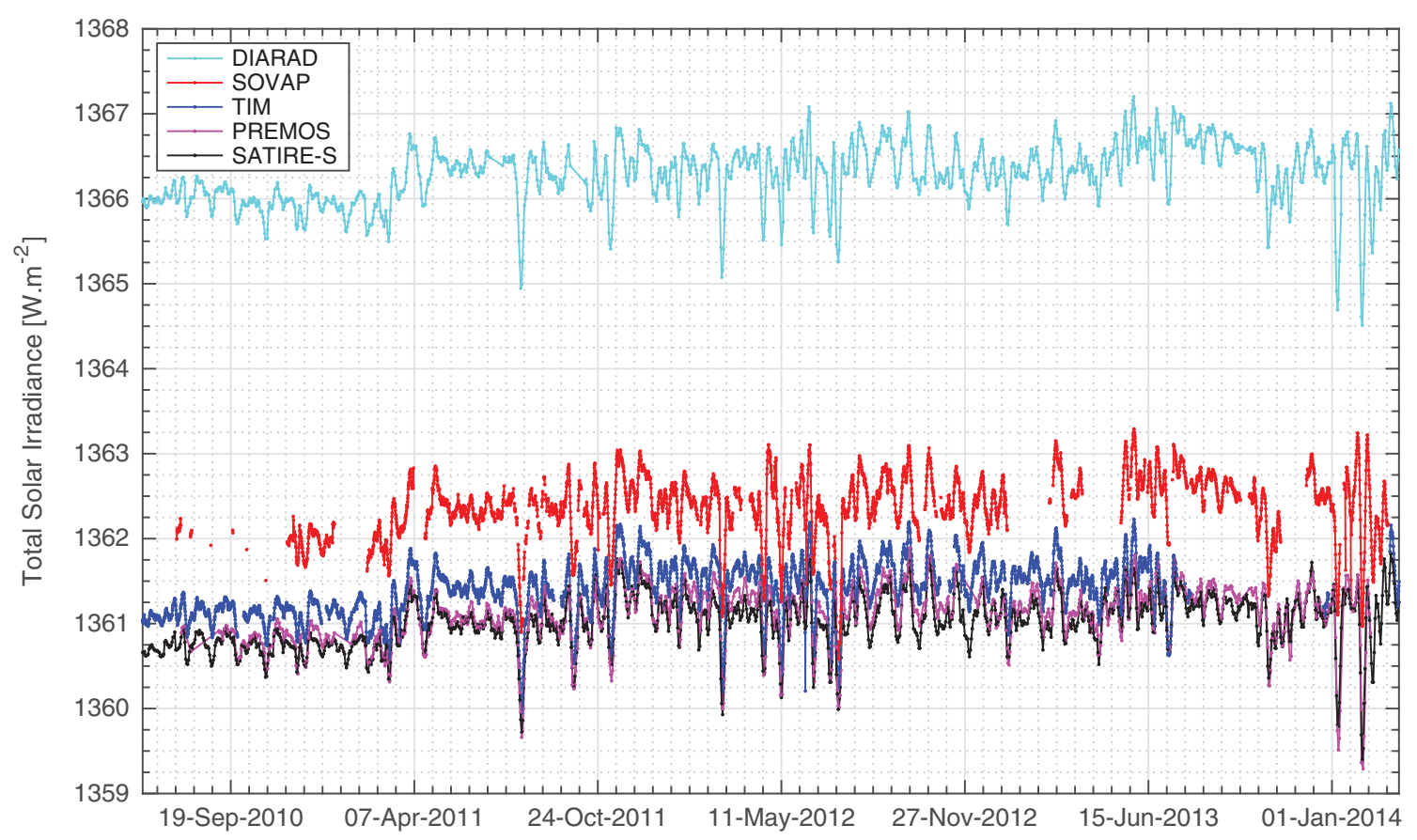

Fig. 4. TSI time series for different space-based TSI radiometers (VIRGO-DIARAD (left channel), SOVAP composite, TIM and PREMOS). The black curve displays the TSI time series of the SATIRE-S semi-empirical model (SATIRE-S has no ability to determine the absolute scale).

(a)

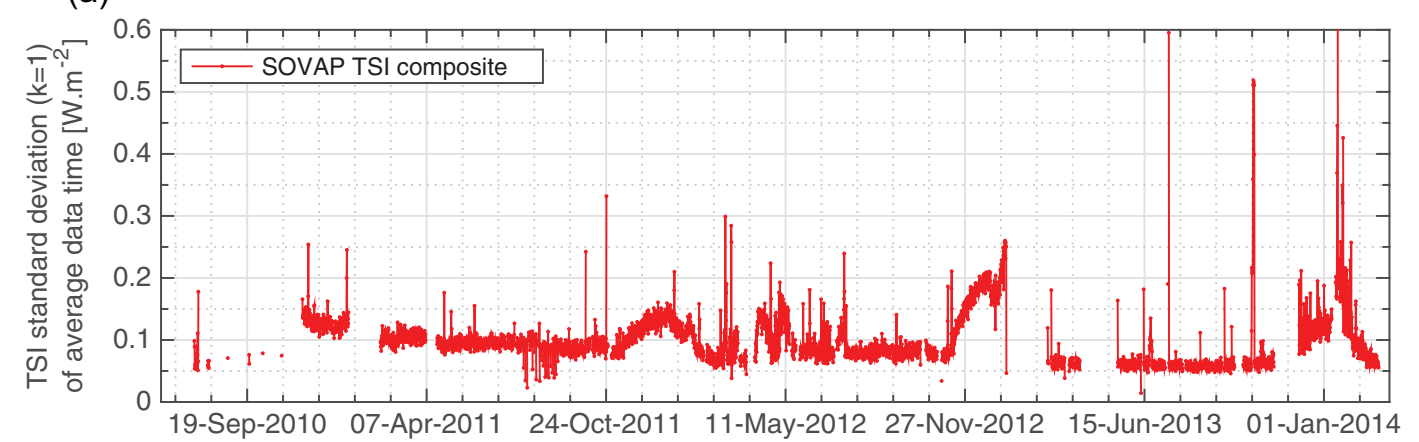

(b)

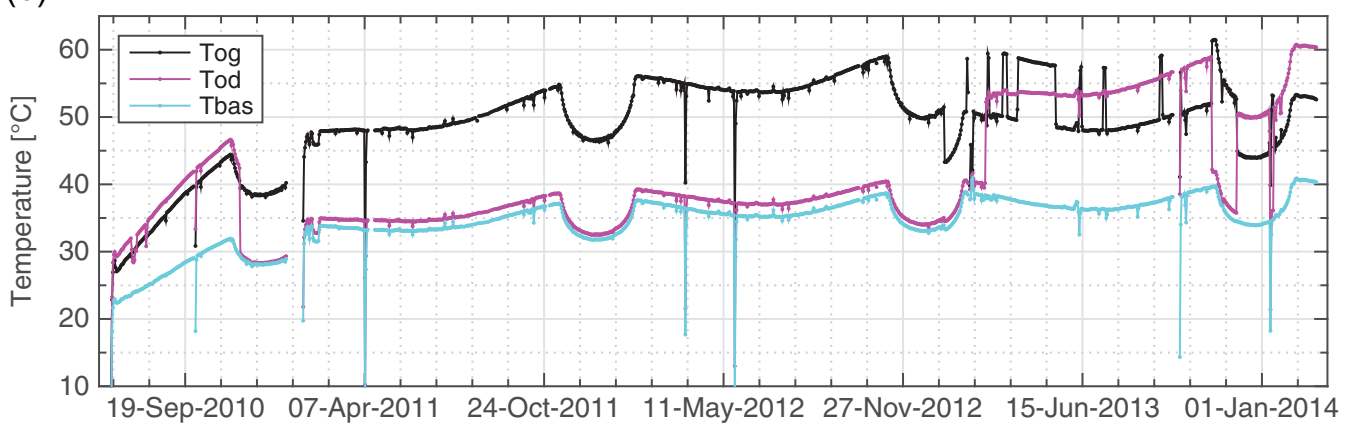

Fig. 5. (a) Standard deviation of average data time (6-h high-cadence data points) of the SOVAP TSI composite. (b) Temperature evolution (daily average) of the main components of the SOVAP radiometer. The black curve displays the temperature evolution of the left shutter (Tog). The magenta curve displays the temperature evolution of the right shutter (Tod). The cyan curve displays the temperature evolution of the radiometric core (Tbas).

The absolute TSI as seen by SOVAP is $1361.8 \pm 2.4 \mathrm{~W} \mathrm{~m}^{-2}$ $(1 \sigma)$ for the 2008 solar minimum period. The new value is significantly lower than DIARAD type values - prior to the launch of TIM - of $1365.4 \mathrm{~W} \mathrm{~m}^{-2}$, but still slightly higher than the TIM $\left(1360.8 \pm 0.5 \mathrm{~W} \mathrm{~m}^{-2}\right)$ or re-calibrated PREMOS and ACRIM values. The DIARAD type radiometer has been kept as an independent absolute radiometer, not calibrated against other radiometers, but only compared to other radiometers for validation. We note that for the SOVAP-DIARAD radiometer, we introduced a new term in the radiometric equation that accounts for the thermoelectrical non-equivalence based on a thermo-electrical model 
Table 3. Pearson's linear correlation coefficient $\left(R^{2}\right)$ between SOVAP, VIRGO-DIARAD, TIM, PREMOS and SATIRE-S TSI time series.

\begin{tabular}{lccccc}
\hline \hline$R^{2}$ linear correlation & $\mathrm{S}$ & $\mathrm{V}$ & $\mathrm{T}$ & $\mathrm{P}$ & $\mathrm{S}-\mathrm{S}$ \\
\hline SOVAP (S) & - & & & & \\
VIRGO-DIARAD (V) & 0.90 & - & & & \\
TIM (T) & 0.76 & 0.67 & - & & \\
PREMOS (P) & 0.88 & 0.88 & 0.78 & - & \\
SATIRE-S (S-S) & 0.92 & 0.92 & 0.78 & 0.93 & - \\
\hline
\end{tabular}

(Meftah et al. 2014). Introducing this term lowered the TSI value of the SOVAP measurements.

\subsection{SOVAP TSI time series}

The determination of the SOVAP TSI time series has required the establishment of modified instrument equations. For the nominal modes, a parameter $\left(\Delta P_{o}\right)$ has been integrated from a theoretical analysis, which highlights the thermo-electrical non-equivalence of the radiometric cavity (open and closed). For the "Rad 10" mode, another parameter $\left(K_{\mathrm{Rad} 10}\right)$ has been integrated from space calibration, which highlights the thermoelectrical non-equivalence between the right and the left cavity. TSI time series for different operation modes of SOVAPDIARAD are displayed in Figure 3 using instrument equations. Thus, continuous time series of TSI observations have been constructed from the set of measurements made by the SOVAP radiometer from the different operation modes. The presented SOVAP composite (Fig. 4) includes all measurement modes normalised to the mean TSI of the "Auto 2" and "Auto 3" modes at the beginning of the PICARD mission.

Three main parameters (thermo-electrical non-equivalence of the active cavity, correction of the thermal radiative effects of the active shutter and efficiency of the left cavity) can vary over time due to ageing. The thermo-electrical nonequivalence of the active cavity may evolve during the mission and the characteristics of the thermo-electric sensor (temperature dependence) and its attachment (thermal contact resistance) may change over time due to space environment. However, this variation is difficult to assess. Thus, the thermo-electrical non-equivalence requires a ground calibration to get an idea of the impact of various parameters and a potential link with SOVAP housekeeping data. Correction of the thermal radiative effects of the active shutter and efficiency of the left cavity parameters depend on the thermo-optical properties (solar absorptance and emissivity) of the SOVAP cavity's coating. The SOVAP cavity is covered with diffuse black paint (Nextel black velvet). Measurements of the reflectance and ageing characteristics $(0.32-14.3 \mu \mathrm{m}$ wavelength range) of common black coatings were performed by Dury et al. (2007). They highlight that the diffuse reflectance remained flat and below $4 \%$ for wavelengths between 0.32 and $2.5 \mu \mathrm{m}$ (solar absorptance is close to 0.96). Moreover, the diffuse reflectance component exhibits a slight increase $(1 \%)$ due to solar ageing (solar absorptance variation is close to 0.01 ). This effect would change the SOVAP TSI measurements by a maximum of $0.5 \mathrm{~W} \mathrm{~m}^{-2}$ over time. Dury et al. (2007) shows that the coating is sensitive to thermal ageing effects (thermal cycling). Physical inspection revealed that the surface of the coating had become cracked after thermal ageing. A representative test of the SOVAP cavity thermal cycling as seen in space may be useful to assess the evolution of the coating. The emissivity of the cavity $\left(15^{\circ} \mathrm{C}\right.$ that corresponds to a wavelength of $10 \mu \mathrm{m}$ ) can also influence the TSI measurements. The emissivity of the SOVAP cavity is close to 0.94 and can vary up to $1.5 \%$ (Dury et al. 2007). $C_{3}$ correction that depends on the emissivity takes into account the contribution of the internal thermal emission of the active shutter and its variation during the mission (less than $0.05 \mathrm{~W} \mathrm{~m}^{-2}$ ). To conclude, it is difficult to have a complete evaluation of the cavity degradation. However, the left channel cavity degradation is very small from February 2013 to March 2014. If we take VIRGO as reference, it is less than $0.1 \mathrm{~W} \mathrm{~m}^{-2}$.

Figure 4 displays the total solar irradiance as seen by the VIRGO-DIARAD radiometer (non-revised by RMIB), SOVAP composite, TIM, PREMOS and SATIRE-S semi-empirical model from July 2010 to March 2014. Time series of SOVAP composite, TIM, PREMOS and SATIRE-S show lower TSI than the canonical value of $1365.4 \pm 1.3 \mathrm{~W} \mathrm{~m}^{-2}$ used a decade ago. The VIRGO TSI measurements (new scale) are consistent with the SOVAP values and highlight the interest to revise the TSI absolute values of all DIARAD type radiometers.

Figure 5a displays the standard deviation of average data time $(6 \mathrm{~h})$ of the SOVAP composite measurements ("Auto 2", "Auto 3" and "Rad 10" modes). Repeatability is less than $\pm 0.1 \mathrm{~W} \mathrm{~m}^{-2}$ and shows the dependence on the temperature of both SOVAP shutters and radiometric core (Fig. 5b). It also highlights the importance of developing cryogenic radiometers to possibly avoid these effects. Scientific requirements of this type of radiometer could provide a TSI-measurement uncertainty (absolute determination) of $\pm 0.5 \mathrm{~W} \mathrm{~m}^{-2}$ ( $\left.\pm 367 \mathrm{ppm}\right)$, a measurement repeatability of $\pm 0.01 \mathrm{~W} \mathrm{~m}^{-2}( \pm 7 \mathrm{ppm})$ and a long-term stability better than $\pm 0.05 \mathrm{~W} \mathrm{~m}^{-2}( \pm 37 \mathrm{ppm})$ during a solar cycle.

\subsection{TSI time series comparisons}

The correlation coefficient $\left(R^{2}\right)$ between SOVAP and the semi-empirical model (SATIRE-S) TSI variations is close to 0.92 . Thus, there is a very good correlation between these two sets of data based on the Pearson definition. All correlations are given in Table 3. The correlation coefficient between SOVAP and TIM observations is less good. All SORCE instruments ceased operations from July 30, 2013 to December 22, 2013 due to an anomaly of the spacecraft (battery degradation). The discontinuity in the TIM data may influence the results.

Figure 6 displays relative differences between SOVAP and each radiometer (VIRGO-DIARAD, TIM and PREMOS) or model (SATIRE-S) TSI variability. Positive or negative trends of the relative differences highlight non-identical behaviours between SOVAP and the considered radiometer. Relative differences between SOVAP and space-based radiometer (VIRGO-DIARAD, TIM and PREMOS) TSI variability are lower than few hundred ppm. Moreover, relative differences between SOVAP and semi-empirical model (SATIRE-S) TSI variability are lower than few hundred ppm (Fig. 6). Between February 2013 and November 2013, negative trends of the relative differences are observed that are less than $80 \mathrm{ppm}$ $\left(\sim 0.1 \mathrm{~W} \mathrm{~m}^{-2}\right)$. During this period, the SOVAP “Auto 2 " mode was operational. Thus, this is probably linked to a degradation of the SOVAP left channel cavity. 

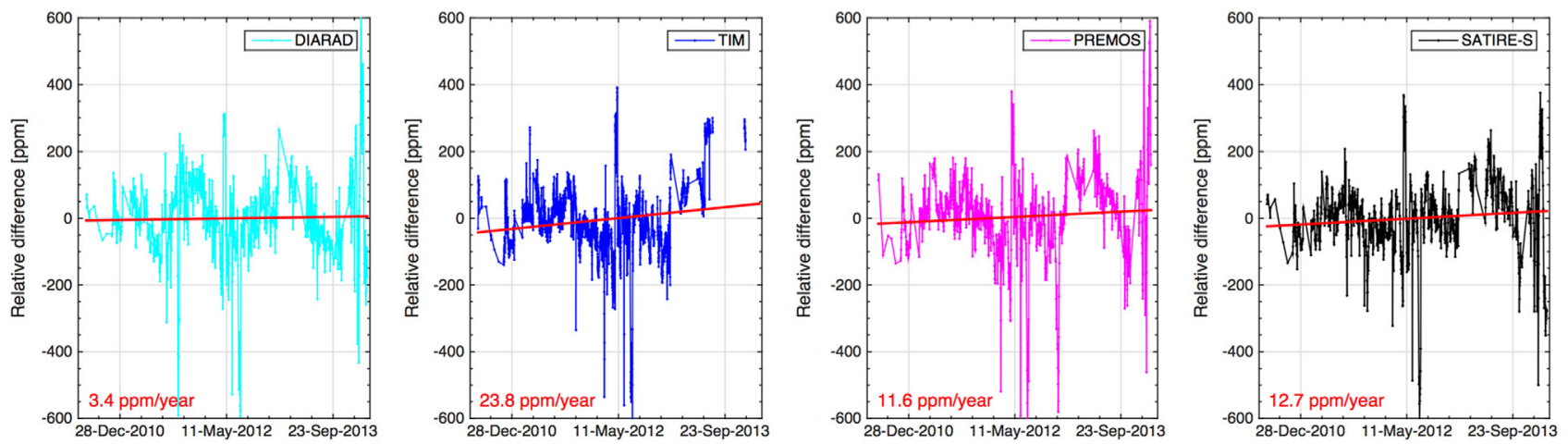

Fig. 6. Relative differences between SOVAP and each instrument (VIRGO-DIARAD, TIM and PREMOS) or model (SATIRE-S) TSI variability. Direction of differences is SOVAP minus instrument or model.

\section{Conclusions}

1. PICARD provides important contributions to TSI variation monitoring during Solar Cycle 24.

2. The SOVAP TSI time series has implemented our stateof-the-art revised instrument equations.

3. SOVAP absolute TSI is equal to $1361.8 \pm 2.4 \mathrm{~W} \mathrm{~m}^{-2}$ $(1 \sigma)$ for the 2008 solar minimum period (VIRGODIARAD TSI data extrapolation).

4. From July 2010 to March 2014, the amplitude of the TSI changes has been of the order of $\pm 0.1 \%$, corresponding to a range of about $2.7 \mathrm{~W} \mathrm{~m}^{-2}$.

5. SOVAP data show very good correlations with VIRGODIARAD and SATIRE-S.

6. The radiometric equations for all other DIARAD type radiometers (and thus the absolute value of the TSI coming from these) should be revised to account of the thermo-electrical non-equivalence.

Acknowledgements. Part of this work was supported by the Centre National de la Recherche Scientifique (CNRS), the RMIB and the Solar-Terrestrial Center of Excellence (STCE). Finally, the authors thank the anonymous referee, Claus Fröhlich and Greg Kopp for many helpful comments and suggestions to improve the manuscript. The editor thanks André Fehlmann and Claus Fröhlich for their assistance in evaluating this paper.

\section{References}

Butler, J.J., B.C. Johnson, J.P. Rice, E.L. Shirley, and R.A. Barnes. Sources of differences in on-orbital total solar irradiance measurements and description of a proposed laboratory intercomparison. J. Res. Natl. Inst. Stand. Technol., 113, 187-203, 2008.

Conscience, C., M. Meftah, A. Chevalier, S. Dewitte, and D. Crommelynck. The space instrument SOVAP of the PICARD mission, in: Society of Photo-Optical Instrumentation Engineers
(SPIE) Conference Series, vol. 8146 of Society of Photo-Optical Instrumentation Engineers (SPIE) Conference Series, 814613, 2011, DOI: $10.1117 / 12.895447$.

Crommelynck, D.A., R.W. Brusa, and V. Domingo. Results of the solar constant experiment onboard Spacelab 1. Sol. Phys., 107, $1-9,1987$

Dewitte, S., D. Crommelynck, and A. Joukoff. Total solar irradiance observations from DIARAD/VIRGO. J. Geophys. Res. [Space Phys.], 109, A02102, 2004, DOI: 10.1029/2002JA009694.

Dury, M.R., T. Theocharous, N. Harrison, N. Fox, and M. Hilton. Common black coatings reflectance and ageing characteristics in the $0.32-14.3 \mu \mathrm{m}$ wavelength range. Opt. Commun., 270, 262-272, 2007, DOI: 10.1016/j.optcom.2006.08.038.

Fröhlich, C., D.A. Crommelynck, C. Wehrli, M. Anklin, S. Dewitte, et al. In-flight performance of the Virgo solar irradiance instruments on SOHO. Sol. Phys., 175, 267-286, 1997, DOI: $10.1023 / \mathrm{A}: 1004929108864$.

Fröhlich, C., J. Romero, H. Roth, C. Wehrli, B.N. Andersen, et al. VIRGO: experiment for helioseismology and solar irradiance monitoring. Sol. Phys., 162, 101-128, 1995, DOI: $10.1007 /$ BF00733428.

Kopp, G., and J.L. Lean. A new, lower value of total solar irradiance: evidence and climate significance. Geophys. Res. Lett., 38, L01706, 2011, DOI: 10.1029/2010GL045777.

Meftah, M., S. Dewitte, A. Irbah, A. Chevalier, C. Conscience, D. Crommelynck, E. Janssen, and S. Mekaoui. SOVAP/Picard, a spaceborne radiometer to measure the total solar irradiance. Sol. Phys., 289, 1885-1899, 2014, DOI: 10.1007/s11207-013-0443-0.

Schmutz, W., A. Fehlmann, W. Finsterle, G. Kopp, and G. Thuillier. Total solar irradiance measurements with PREMOS/PICARD, in: American Institute of Physics Conference Series, vol. 1531 of American Institute of Physics Conference Series, 624-627, 2013, DOI: $10.1063 / 1.4804847$.

Willson, R.C. ACRIM3 and the Total Solar Irradiance database. Astrophys. Space Sci., 352, 341-352, 2014, DOI: $10.1007 / \mathrm{s} 10509-014-1961-4$

Yeo, K.L., N.A. Krivova, S.K. Solanki, and K.H. Glassmeier. Reconstruction of total and spectral solar irradiance from 1974 to 2013 based on KPVT, SoHO/MDI, and SDO/HMI observations. $A \& A, \mathbf{5 7 0}, \mathrm{A} 85,2014$, DOI: 10.1051/0004-6361/201423628.

Cite this article as: Meftah M, Chevalier A, Conscience C \& Nevens S. Total solar irradiance as measured by the SOVAP radiometer onboard PICARD. J. Space Weather Space Clim., 6, A34, 2016, DOI: 10.1051/swsc/2016027. 